\title{
Primary Care Transformation and Physician Burnout
}

\author{
Karin Nelson, MD, MSHS ${ }^{1,2}$ and Greg Stewart, $\mathrm{PhD}^{3,4}$ \\ 'VA Puget Sound Health Care System, Seattle, WA, USA; ${ }^{2}$ Department of Medicine, University of Washington, Seattle, WA, USA; ${ }^{3}$ VA lowa City \\ Health Care System, lowa City, IA, USA; ${ }^{4}$ Department of Management, University of lowa, lowa City, IA, USA.
}

J Gen Intern Med 34(1):7-8

DOI: $10.1007 / \mathrm{s} 11606-018-4722-1$

(c) Society of General Internal Medicine (This is a U.S. government work and not under copyright protection in the U.S.; foreign copyright protection may apply) 2018

${ }^{66} \mathrm{~F}$ irst, do no harm" is an oft-quoted adage regarding
physicians' obligation to help patients in ways that do not end up making things worse. Should not the same principle apply to organizational leaders attempting to improve health care delivery systems? What if transformation initiatives designed to improve patient care create additional stress that negatively impacts the work experience and thereby ends up degrading patient care? Such questions are frequently asked by both leaders and front-line participants of organizations encountering increased pressure to transform health care delivery to reduce costs and improve care.

A pervasive indicator of ongoing stress for physicians and other health care professionals is burnout, which encompasses feelings of exhaustion, and is experienced by 30 to $50 \%$ of practicing physicians. ${ }^{1}$ Specific components of burnout include emotional exhaustion, depersonalization, and a decreased sense of personal accomplishment. These negative feelings have important organizational consequences, including provider turnover and worse patient outcomes. ${ }^{2}$ Improving the physician work experience by reducing negative feelings such as burnout seems a worthy pursuit in and of itself. Indeed, assuring a positive provider work experience has been proposed as the quadruple aim in health care; along with the triple aim of enhancing patient experience, improving population health, and reducing costs. ${ }^{3}$ Given that burnout often accompanies feelings of having too much to do and being pulled in multiple different directions, it seems at least plausible that extra work created by well-intended transformation initiatives designed to make things better for both patients and health care providers may actually have the unintended consequence of making things worse.

In this issue, the study "The Effects of a Primary Care Transformation Initiative on Primary Care Physician Burnout and Workplace Experience" by Peikes and colleagues examines the effect of participation in a large-scale health care transformation initiative - the Comprehensive Primary Care (CPC) program —on the physician work experience, with an emphasis on burnout. ${ }^{4}$ CPC is a multi-payer primary care

Published online November 7, 2018 transformation initiative instituted by Medicare from October 2012 through December 2016. CPC specifically required care delivery changes and provided enhanced payment, data feedback, and learning support. The authors obtained survey responses from over 1000 physicians, and compared responses from those participating in the $\mathrm{CPC}$ practices to a matched group of others not participating in the CPC program. Although a high percentage of physicians reported feeling burned out, there was no evidence that responses from those involved in the CPC initiative differed meaningfully from responses provided by the control group.

There are several possible explanations why the current study did not identify differences in burnout related to participation in the CPC. Perhaps the most plausible reason is that the transformation initiative was simply not designed to decrease burnout as a primary outcome. Specifically, three areas have been consistently associated with burnout: loss of autonomy and low perceived control, lack of meaning in work, and working in inefficient systems with excessive workload. ${ }^{5}$ Other drivers of burnout include a high burden of responsibility, discordance between individual and organizational values, unsupportive work environments, and isolation. ${ }^{6}$ Although CPC did not focus specifically on any of these critical elements, practice facilitation potentially improved the work environment along some of these lines. At the same time, the CPC intervention certainly created the need for organizational change and thereby likely at least temporarily decreased feelings of control and inefficiency. Perhaps these competing pressures simply acted as offsets, resulting in little or no difference in the work environment of health care providers.

From the optimist perspective, it is good to see that physician burnout was not higher in CPC practices compared to other practices. Overall, the results of this study add to the growing body of knowledge making it clear that a considerable number of physicians feel burned out, and that transforming health care delivery in ways that improve working conditions and health outcomes is challenging. Although early evaluations of transformation initiatives such as the medical home model and teambased approaches to care delivery found some evidence of decreased burnout, ${ }^{7}$ data since then has been mixed and little support has been garnered for transformation initiatives that universally decrease physician burnout.

Even interventions designed specifically to decrease burnout are often linked only to moderate improvement. ${ }^{8}$ Most studies of burnout suggest that both an individual and 
organization-level approach are needed. ${ }^{9}$ A recent metaanalysis of burnout intervention found that few interventions focused specifically on primary care practice, and that interventions which focused more on organizational-directed, as opposed to physician-directed, were most effective. ${ }^{8}$ It may not be the content of the transformation intervention itself that matters but rather the effectiveness with which the transformation is implemented. In other words, simply engaging or not engaging in a transformation initiative may have little impact on perceptions such as burnout; however, the way that the initiative is carried out may make all the difference.

Our own work evaluating the effectiveness of a large-scale health care delivery transformation in Veterans Health Administration has led us to identify specific aspects of how a transformation is implemented that significantly impact the work experiences of front-line physicians and other employees. ${ }^{10}$ Our work also points to the high rates of burnout in clinical staff in addition to physicians, including nurse practitioners, nurses and clerical staff. Yet, burnout is reduced under three conditions: guidance by supportive leadership, provision of adequate resources, and facilitation of effective teamwork. ${ }^{10,11}$ Supportive leaders ensure open communication and engage front-line providers and other staff in transformation initiatives by integrating new practices with ongoing clinic procedures. One of the most critical resource these leaders provide is adequate staff that helps assure those carrying out the transformation are not overburdened by the need not only to provide care to patients but also to learn new skills and implement change. Developing stable teams that work together and support one another to share the burdens of implementing change also helps reduce individual stress and change fatigue. In short, successful implementation of a transformation initiative requires significant time and effort, and those charged with carrying out changes experience stress and burnout when new burdens are added to ongoing duties without additional time and resources to learn and enact new behaviors. Thus, organizations need to consider the work experience of physicians and other primary care team members as they seek to transform health care delivery. When effects on the well-being and engagement of healthcare workers are not taken into account, a likely result is frustration and quite possibly an initiative that ends up making things worse.

Corresponding Author: Karin Nelson, MD, MSHS; VA Puget Sound Health Care System, Seattle, WA, USA (e-mail: Karin.Nelson@va.gov).

\section{Compliance with Ethical Standards:}

Conflict of Interest: We have no conflicts of interest to report.

\section{REFERENCES}

1. Shanafelt TD, Hasan O, Dyrbye LN, et al. Changes in Burnout and Satisfaction With Work-Life Balance in Physicians and the General US Working Population Between 2011 and 2014. Mayo Clin Proc. 2015;90(12):1600-1613.

2. Dyrbye, L.N., T.D. Shanafelt, C.A. Sinsky, P.F. Cipriano, J. Bhatt, A. Ommaya, C.P. West, and D. Meyers. 2017. Burnout among health care professionals: A call to explore and address this underrecognized threat to safe, high-quality care. NAM Perspectives. Discussion Paper, National Academy of Medicine, Washington, DC.

3. Bodenheimer T, Sinsky C. From triple to quadruple aim: care of the patient requires care of the provider. Ann Fam Med. 2014;12(6):573-576.

4. Peikes DN, Swankoski K, Hoag SD, et al. The Effects of a Primary Care Transformation Initiative on Primary Care Physician Burnout and Workplace Experience. J Gen Intern Med. 2018; https://doi.org/10. 1007/s11606-018-4545-0.

5. Shanafelt TD, Dyrbye LN, West CP. Addressing Physician Burnout: The Way Forward. JAMA. 2017;317(9):901-902.

6. Epstein RM, Privitera MR. Doing something about physician burnout. Lancet. 2016;388(10057):2216-2217.

7. Reid RJ, Coleman K, Johnson EA, et al. The Group Health medical home at year two: cost savings, higher patient satisfaction, and less burnout for providers. Health Aff (Millwood). 2010;29(5):835-843.

8. Panagioti $\mathbf{M}$, Panagopoulou E, Bower $\mathbf{P}$, et al. Controlled Interventions to Reduce Burnout in Physicians: A Systematic Review and Metaanalysis. JAMA internal medicine. 2017;177(2):195-205.

9. West CP, Dyrbye LN, Erwin PJ, Shanafelt TD. Interventions to prevent and reduce physician burnout: a systematic review and meta-analysis. Lancet. 2016;388(10057):2272-2281.

10. Helfrich CD, Simonetti JA, Clinton WL, et al. The Association of TeamSpecific Workload and Staffing with Odds of Burnout Among VA Primary Care Team Members. J Gen Intern Med. 2017;32(7):760-766.

11. Helfrich CD, Dolan ED, Simonetti J, et al. Elements of team-based care in a patient-centered medical home are associated with lower burnout among VA primary care employees. Journal of general internal medicine. 2014;29(2):659-666. 